\title{
Immunological Pathways in Hidradenitis Suppurativa: Current Concepts and Innovative Therapies
}

\author{
José Antonio Guzmán Cubilla ${ }^{{ }^{*}}$, Beatrice Martinez Zugaib Abdalla ${ }^{2}$, Paulo Ricardo Criado ${ }^{1}$ and Luiza \\ Keiko Oyafuso ${ }^{1}$ \\ ${ }^{1}$ Dermatology, ABC School of Medicine, Santo André, SP, Brazil \\ ${ }^{2}$ General Medicine, ABC School of Medicine, Santo André, SP, Brazil
}

Received: April 04, 2018; Accepted: April 17, 2018 ; Published: April 23, 2018

*Corresponding author: José Antonio Guzmán Cubilla, Rua da Fonte 77, APTO 163, Santo André, SP, Brazil, Zip code: $09040270 ;$ Tel: +55 11 982060707; E-mail: joseantoniojuly89@gmail.com

\begin{abstract}
Hidradenitis Suppurativa is a chronic and disabling disease characterized by pustules, nodules, abscesses, and scaring. It affects natural body folds (inguinal, axillary, inframammary), perianal region and genitalia. Several comorbidities are related to this disease, such as: obesity, smoking, acne, folliculitis, and hyperandrogenism. Disorders in keratinization, systemic upregulation of complement (C5a) activation and changes in cutaneous microbiome stimulate the development of autoinflammatory state, promoting the increase of CD4+ T-helper lymphocytes (Th-17 and Th-1). These changes stimulate the expression of toll-like receptors type 2, enabling the NLRP3 inflammasome and triggering of the caspase-dependent inflammatory cascade. Intralesional increase of dendritic cells, macrophage and neutrophil influx stimulate the production of pus, promoting necrosis and apoptosis of inflammatory cells undergoing a special type of cell death program, releasing proinflammatory cytokines (pyroptosis). In addition, patients affected with this condition presents an imbalance of IL-36 proinflammatory cytokines and decreased levels of its antagonist receptor IL-36Ra. Treatment is frustrating and includes the use of broad-spectrum antibiotics, surgical procedures, and oral retinoids. The first and only one biologic therapy approved is a TNF- $\alpha$ inhibitor (Adalimumab); other alternatives are under development, inhibiting IL-17 (Secukinumab, Bimekizumab and CJM112) IL-12 and IL-23 (Ustekinumab); and IL-1 (Anakinra, Canakinumab). Recent articles showed that liraglutide, a glucagon-like-1 pep-tide (GLP-1) agonist, could be used to treat one of the comorbidities associated with HS (insulin resistance). Finally, inhibition of IL-36 and C5a could be an option to treat this recalcitrant and exhausting disease.
\end{abstract}

Highlights: Pathogenesis of HS involvesgenetic and environmental factors that trigger the innate immune response.

Several cytokines participate in the inflammatory response of the skin.

HS is chronic and relapsing causing a negative impact on patient's quality of life, expensive medical approaches, and huge number of comorbidities.

New biological drugs, former medical treatments and cutaneous surgical interventions can be combined to better assist of patients suffering of HS.

Keywords: Hidradenitis suppurativa; Biologics; IL-17; IL-1; IL23; C5a; Autoinflammatory diseases;

\section{Introduction}

Hidradenitis Suppurativa (HS), also known as "acne inversa" or "maladie de Verneuil,", is a rare inflammatory skin disorder, about $0.035 \%$ to $4 \%$ of the world population suffer from this condition, mostly affecting adults and adolescents with few reported cases in prepubertal children [1,2]. Disease course is recurrent or intermittent and chronic, with multiple clinical variants, a 3:1 female-male ratio, and apparently without ethnic preference [2]. However, Afro-descendants seem to develop more exuberant lesions than Caucasians patients. Usually the onset is puberty-related, suggesting an endocrine component and associated with increased levels of androgenic hormones [2].

Due to a delay in diagnosis and lack of awareness by physicians, final diagnostic is achieved after an average of 12 years [2]. Also, HS patients score high values in the Dermatology Quality of Life Index (DLQI), with higher values than the average found in psoriasis [3]. High values in this score are related to a greater impact in patient's life [3].

Heterogeneous mutation in the intramembranous protease complex $\gamma$-secretase and its subunits genes NCSTN, PSENEN, and PSEN1 has been associated with familial inheritance of HS. These mutations have been associated with impaired inhibition of the innate immunity by the Notch-MKP-1 signaling, promoting cutaneous proinflammatory microenvironment, inducing follicular keratinization and epidermal hyperplasia. Furthermore, mutations of $y$-secretase complex have also been implicated in Alzheimer's Disease (AD); however, no link between HS and AD has been documented. About $42 \%$ of patients report family history, early onset at puberty and more severe disease manifestations [4]. In addition, mutations in PSENEN are found to be present in patients with Dowling-Degos Disease (DDD). Around half of patients with DDD develop HS and share similar trigger factors [5].

\section{Discussion}

\section{Clinical Features}

HS is characterized by papules, nodules, abscesses, and painful 
suppurating sinus, these lesions can progress into fibrosclerotic plaques, keloids, or atrophic scars, decreasing and disabling the patient movement [6]. Inguinal, axillary, inter gluteal and inframammary folds are predominantly affected, however, ectopic locations such as the head, torso, genitals, dorsum, and limbs are uncommonly found in some patients [6]. Chronic and advanced cases may develop lower limb and genitalia lymphedema, as well as, hinder social and sexual relationships [7]. The disease has a recurrent course with an average of 2 to 3 crises every 6 months [2].

Diagnosis of HS is often clinical. Cutaneous biopsies are not required in most cases [2]. There are several scoring systems for assessing disease severity: Hurley Staging System (HSS), HSPhysician's Global Assessment, Modified Sartorius Score (MSS), and Hidradenitis Suppurativa Severity Index). Unfortunately, all of them have some limitations in daily practice, with no consensus among the outcome measures used for HS [2].

HSS and MSS are the most widely applied disease activity scores [2]. Hurley scoring is usually stratified into three stages [2]. Stage I: single or multiple abscess, without scars and sinus tracts formation. Stage II: recurrent single or multiple abscess with sinus tracts and scars formation, lesions are widely separated. Stage III: diffuse involvement or multiple interconnected tracts and abscesses formation across entire area.

Smoking, obesity, hyperandrogenism and women with Polycystic Ovarian Syndrome (PCOS) seem be related to HS, worsening the disease evolution [8].

Remarkably, the presence of other associated diseases such as acne, pyoderma gangrenosum, neutrophilic diseases, Crohn's disease, pilonidal cyst, spondyloarthropathies and psoriatic arthritis are commonly seen in some patients. These conditions could be overlapping and develop syndromes such as the SAPHO (Synovitis, Acne, Pustulosis, Hyperostosis and Osteitis), PASH (Pyoderma gangrenosum, Acne and Hidradenitis suppurativa), PAPA (Pyogenic Arthritis, Acne, and Hidradenitis suppurativa) and PAPASH (Arthritis, Pyoderma, Acne, and Hidradenitis suppurativa) [8].

Due to its polymorphism, multiple differential diagnoses are included, such as lymphogranuloma venereum, donovanosis, tertiary syphilis, paracoccidioidomycosis, furunculosis, atypical mycobacterial and tuberculosis infection with abscesses, metastatic Crohn's disease and anal fistulas. Delayed diagnosis is common and usually done by an experienced physician [8].

\section{Pathophysiology}

The pathogenesis of HS until recent years was still poorly understood. Because lesions are localized in body folds areas such as the axilla, groin, perineum, and genitalia, it was long thought to be a primary disorder of the apocrine glands [9]. During the last decade, several evidences clarified that the primary defect in HS pathophysiology involves occlusion and subsequent inflammation of the hair follicle [9]. Morphological changes in the pilosebaceous unit plays a critical role in the development of HS, these follicular changes are characterized by an hyperkeratinization of the follicle infundibulum associated to defects in keratin proteins production (decreased number of cytokeratin CK17, with upregulated CK5 and CK6) $[10,11]$. This suggests fragility of the draining sinus epithelium, which may allow rupture to occur more easily, thus resulting in a subcutaneous abscess formation. Blocked follicles modify the skin microbiome and increases bacterial proliferation (S. epidermidis and P. acnes), generating a film-like substance that remain fixed to the follicular unit (bacterial biofilm), and stimulating skin overexpressed Toll-like receptors type 2 (TLR2) [12-15].

TLR2 is a vital component of the innate immunity, present in polymorphonuclear cells, keratinocytes, and dendritic cells. Fragments of bacterial antigens called PAMPS (Molecular patterns associated with pathogens) activate this receptor and initiate skin inflammation.

Activation of TLR2, increase intralesional expression of endogenous antibiotics called antimicrobial Peptides (AMPs), such as is human beta defensin-2 (HBD2) and cathelicidin LL37 (LL37) [15]. These peptides interfere further in the inflammatory process and induce hyperkeratinization of the hair follicle, triggering the formation of the NLRP3 inflammasome and of the pro inflammatory cytokines (IL-1 $\beta$, IL-12, IL-23, IL-17, and TNF- $\alpha$ ).

NLRP3 inflammasome activates the caspase inflammatory cascade, increasing caspase- 1 expression, this enzyme plays an important role in phagocytes tissue migration, promoting polymorphonuclear cells death in presences of newly formed interleukin-1beta (IL-1 $\beta$ ) by a process called pyroptosis (cell death with release of inflammatory cytokines) [16].

This process leads to cellular DNA damage and rapid pore formation in dendritic and macrophages cells membrane, resulting in cellular lysis and release of intracellular inflammatory contents, including Interleukin-1beta and alpha (IL-1 $\beta$, IL-1 $\alpha$ ) [17]. Excess release of IL- $1 \alpha$ from the cytosol of necrotic and stressed-cells leads to the recruitment of hematopoietic cells that can further sustain perilesional inflammation through endothelial activation and disruption of vascular walls [17].

Neutrophils migrate into the damaged skin and undergo phagocytosis and cellular death, forming networks of extracellular fibers mainly composed by neutrophilic nuclear and granular contents such as elastase, myeloperoxidase, and DNA, binding to pathogens fragments (NETosis) [18]. This process further stimulates the expression of interleukins, especially IL-8, TNF- $\alpha$ and IL-17; promoting chemotaxis of new neutrophils into the affected skin, pus formation and continuing the inflammatory loop [19].

Recent studies indicate that intralesional and serological increase of Th-17 T-helper lymphocytes and its cytokine (IL-17 and IL-22) may be a significant risk factor for the development of HS [20]. Patients with metabolic syndrome, smokers and Polycystic Ovary Syndrome (PCOS) have a considerable serological augmentation in both IL-17 and TNF- $\alpha$ [21-24]. These systemic alterations could explain the link between these comorbidities with HS [19]. 
Hyperandrogenism, puberty and PCOS stimulate the production of androgen hormones, increasing follicular hyperkeratosis and sebum production; these alterations clog the pore, generating more inflammation $[24,25]$. Besides the systemic inflammatory effect, obesity generates friction in the affected body folds, worsening the follicular obstruction [26]. Remarkably, smoking also increase inflammatory cytokines and stimulate skin cholinergic receptors, these alterations will increase follicular keratinization and neutrophil migration [22].

Recent publications have shown an increased expression of

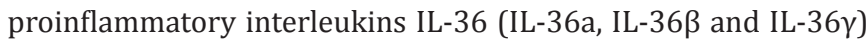
and decreased antagonist cytokines (IL-36Ra, IL-37, IL-38) in the lesional and perilesional skin of patients with HS [27].

Pro-inflammatory IL-36 are members of the interleukin-1 (IL-1) superfamily, involved in the inflammasome activation, and inducing pro-inflammatory signaling pathways through the activation of the Nuclear Factor-kB (NF-kB) and Mitogen Activated Protein Kinase (MAPK) [27]. These cytokines may have an important role in the onset and regulation of the inflammatory response, especially in those patients with autoimmune and autoinflammatory diseases, including pustular psoriasis, rheumatoid arthritis, and inflammatory bowel diseases [28-30].
In HS, IL-36 increase Dendritic Cells (DC) activation, neutrophils influx, keratinocytes proliferation, and secretion of proinflammatory factors, (IL-1ß, TNF- $\alpha$, IL-6, IL-8), stimulating the production of T- helper lymphocytes type 1 (Th1) and Th17, and its cytokines (IL-17, IL-22 and IL-23). This loop perpetuates the autoinflammatory process [31].

Patients suffering of HS present decreased expression of the IL-36 receptor antagonist (IL-36Ra). This antagonist is commonly expressed in dendritic cells, lymphocytes, and keratinocytes, inhibiting the union of IL-36 with its peripheral receptor (IL36R), regulating the inflammatory process in healthy patients [28]. Also, immunosuppressive cytokine (IL-10) seen to be upregulated in affected skin as a compensatory response to the pro inflammatory process. However, this augmentation suppresses only IL-12 with no modification in IL-17 lesional levels. Lower levels of IL-22 induce a decreased formation of AMP compared to psoriasis; maybe this could explain the defected skin antimicrobial function in HS [8].

Recently, Kanni et al. demonstrated that systemic complement activation is up-regulated in HS patients [32]. Elevated C5a and membrane attack complex (C5b-9) induce the expression of TNF- $\alpha$ by circulating mononuclear cells, contributing to the lesional chemotaxis and infiltration of neutrophils [32]. (Figure 1)

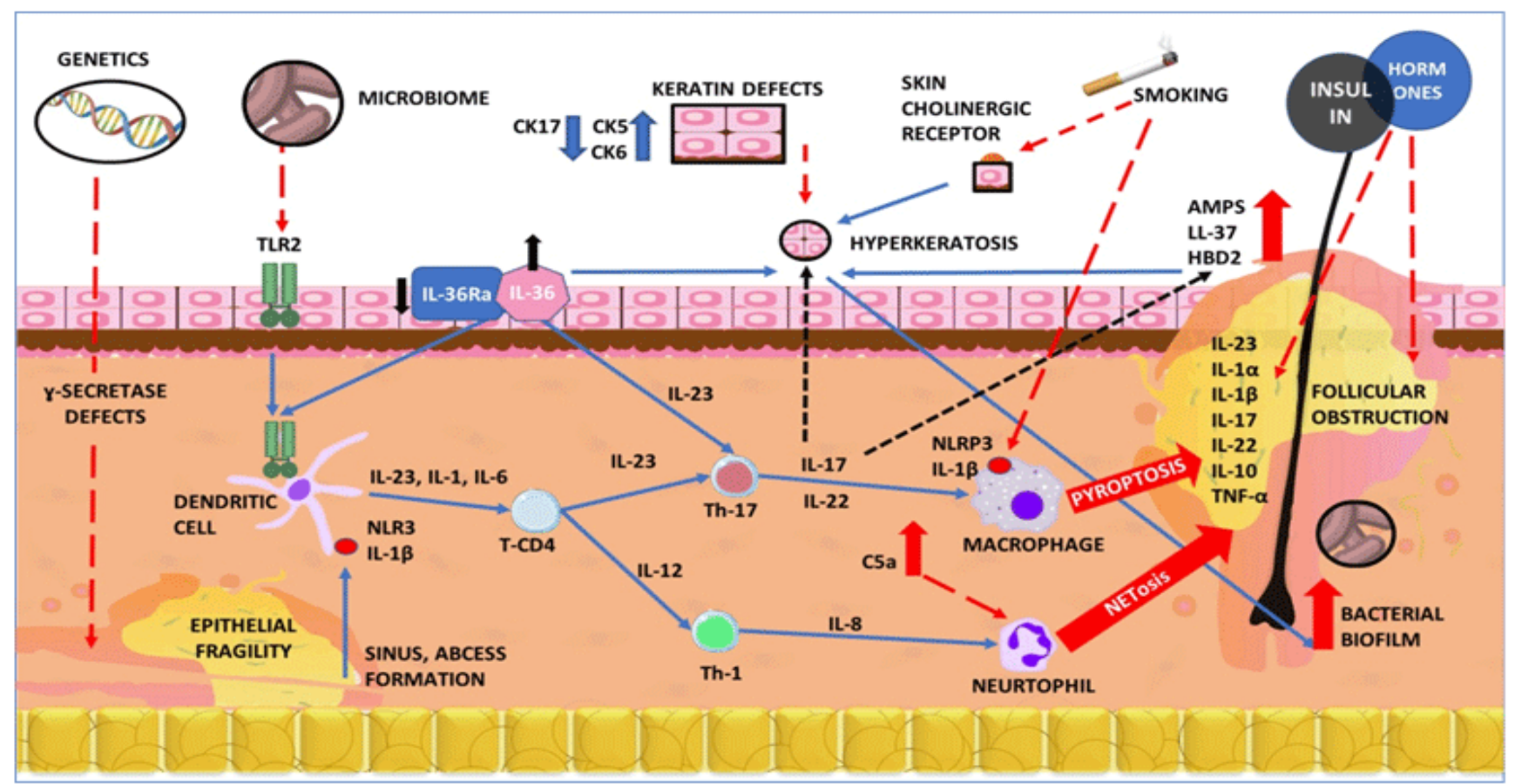

Figure 1: Pathogenesis of Hidradenitis Suppurativa

Genetic defects in the y-SECRETASE subunits promote skin epithelial fragility inducing sinus and abscess formation. Alterations in skin microbiome induce cutaneous and inflammatory cells overexpression of TLR2 promoting a pro inflammatory status. Increase number of IL-36 and decrease of IL-36Ra result in hyperkeratosis and lesional inflammation. Keratin defects promote infundibular hyperkeratosis and fragility, increasing bacterial biofilm, these lesional effects promotes LL37, HBD2 formation acting as a positive feedback for inflammation and follicular obstruction. Smoking promotes activation of NLRP3 with neutrophils and macrophages increase influx, these cells will suffer NETosis and pyroptosis, releasing inflammatory cytokine into the follicular unit and perilesional skin. Nicotine stimulates skin cholinergic receptors inducing hyperkeratosis and follicular obstruction. Hormones and high insulin levels also induce follicular obstruction and cytokines (IL-17, IL-23, IL-1 and TNF $\alpha$ ) release. Systemic Complement activation (C5a) contributes in even more neutrophil migration and stimulates the production of TNF- $\alpha$. 


\section{Therapeutic Approaches}

Hidradenitis suppurativa is a difficult-to-treat disease. Due to smoking and obesity show the strongest correlation with disease severity, there is a consensus that patients suffering for HS must be advised and supported in the cessation of tobacco use and in reducing their body weight [2].

For long time, patients with mild and intermediate stages (Hurley I-II) undergo clinical treatment consisted of oral broad-spectrum antibiotics (Doxycycline, Trimethoprim-
-Sulfamethoxazole, Clindamycin), oral retinoids (Acitretin, Isotretinoin), dapsone, 15\% topical resorcinol and small surgical procedures (Deroofing, laser, and local excision) [2].

In severe stages (Hurley III) the treatment is based on wide excision surgeries, forming deformed scars, with high rate of recurrence after procedures $[2,33]$.

On table 1, we summarized the actual and new promising biological drugs studied to treat HS in distinct points of it pathophysiology.

Table 1: Actual, novel, and future biological drugs for the treatment of hidradenitis suppurativa

\begin{tabular}{|c|c|c|c|}
\hline Name & Target & Clinical trial & Administration \\
\hline Adalimumab & TNF- $\alpha$ & Approved & Subcutaneous \\
\hline Etanercept & TNF- $\alpha$ & II & Subcutaneous \\
\hline Infliximab & TNF- $\alpha$ & II & Intravenous \\
\hline Anakinra & IL-1 antagonist receptor & II & Subcutaneous \\
\hline MABp1 & IL-1 $\alpha$ & II & Intravenous \\
\hline Ustekinumab & $\begin{array}{l}\text { IL-12 e IL-23 from p40 } \\
\text { subunits }\end{array}$ & II & Subcutaneous \\
\hline Secukinumab & IL-17A & I & Subcutaneous \\
\hline Bimekizumab & IL-17, IL-17F & II & Subcutaneous and Intravenous \\
\hline Liraglutide & GLP-1 agonist & Off label, approved for type 2 diabetes & Subcutaneous \\
\hline ANB019 & IL-36R & $\begin{array}{l}\text { I, in a study for generalized pustular } \\
\text { psoriasis }\end{array}$ & Subcutaneous and Intravenous \\
\hline IFX-1 & $\mathrm{C} 5 \mathrm{a}$ & II & Intravenous \\
\hline
\end{tabular}

\section{TNF- $\alpha$ Inhibitors}

With the development of monoclonal antibody-based therapy, the first approved immunobiological for HS was launched, adalimumab, a human monoclonal antibody capable of blocking TNF- $\alpha$ improving lesions and patient's quality of life after 12 weeks of treatment [34]. In some patients, early initiation of this therapy combined with surgery may result in a decrease in outcome and deforming lesions [35]. Adalimumab is given with an initial subcutaneous dose of $160 \mathrm{mg}$ on day 0 of treatment, then $80 \mathrm{mg}$ on day 14 , and lastly $40 \mathrm{mg}$ per week from day 29 of treatment [34]. Other anti-TNF- $\alpha$ agents such as infliximab or etanercept have only reached Phase II clinical studies and failed to prove to be more effective than adalimumab [36-38].

\section{IL-12 and IL-23 Inhibitors}

Ustekinumab is a human monoclonal antibody that acts against the $\mathrm{p} 40$ subunit of interleukins IL-12 and IL-23. Approved for use in psoriasis and Crohn's disease, it was used in an open study where $17 \mathrm{HS}$ patients participated, achieving 12 of them a good response after 40 weeks of treatment [39]. However, more clinical studies are needed to prove the efficacy and safety of this drug in HS. Three new selective p19 subunit inhibitors are currently in phase III clinical trials, guselkumab, risankizumab, and tildrakizumab, preliminary results have shown greater efficacy than ustekinumab in psoriatic patients [40]. However, the use of them in HS is still not being studied; moreover, it would be interesting to do a clinical study focused on this condition.

\section{IL-1 Inhibitors}

Anakinra is a recombinant IL-1 receptor antagonist (IL-1Ra) used in patients with rheumatoid arthritis. In a study involving 12 HS patients, 10 of them achieved considerable results after 12 weeks of treatment [41]. However, there are reports of isolated cases with no therapeutic benefits [42].

Canakinumab is a monoclonal antibody against IL-1 $\beta$, useful in autoimmune diseases [43]. Several reports have shown promising results in patients with HS. However, there is no extensive study proving its efficacy.

MABp1 is a recombinant human IgG1 monoclonal antibody specific for human interleukin- $1 \alpha$. The mechanism of action is thought to be the inhibition of neovascularization and modulation of the production of IL- 8 and human $\beta$-defensin- 2 . A randomized study with 20 patients, has recently viewed this drug as a promising agent for HS, including those not eligible for treatment with adalimumab [44]. 


\section{IL-17 Inhibitors}

Secukinumab is a monoclonal antibody against IL17A, with approved use for moderate to severe psoriasis, there are 2 cases with HS treated with satisfactory results and currently the originator is recruiting HS patients for new clinical trials [45-47]. Two new monoclonal antibodies against IL-17, bimekizumab and CJM11243, are being evaluated as possible treatment in patients with HS, yet without preliminary results $[48,49]$.

\section{Incretins}

Liraglutide is a glucagon-like-1 peptide agonist (GLP-1). Used in type 2 diabetes mellitus, when diet and exercise alone are not sufficient for glycemic control. It is responsible for decreasing systemic and peripheral insulin resistance, recently used in 2 cases with HS, improving DLQI and modifying one of the comorbidities associated with HS [50].

\section{IL-36 Inhibitors}

AnaptysBio Inc. announced positive results in a phase I trial evaluating a new drug (ANB019), an anti-interleukin-36 (IL-36R) antibody [51]. Obtained data demonstrated pharmacokinetic and pharmacodynamic safety and may now continue phase II clinical research in patients with palmoplantar and generalized pustular psoriasis [51]. As previously described, in HS there is an increase of this interleukin. Although these drugs are still in development, soon they may be a promising therapeutic option against this chronic and recalcitrant condition.

\section{C5a Inhibitors}

In an open label clinical phase II Study, a novel C5aspecific monoclonal antibody (IFX-1) effectively neutralized the overproduced C5a in HS patients. IFX-1 was tested in 12 patients with moderate to severe HS. All patients received nine intravenous doses of $800 \mathrm{mg}$ IFX-1 on days $1,4,8,15,22,29,36$, 43 and 50. Plasma level of C5a was determined before the trial, at days 22 and 50 during the treatment with IFX-1, with a significant decrease of C5a levels the end of study [52].

Hidradenitis Suppurativa Clinical Response (HiSCR) was applied to evaluate clinical improvement to treatment, achieving up to $83 \%$ response rate at the endpoint of the study. This novel drug may represent an effective treatment for HS patients [52].

\section{Conclusion}

In summary, HS is a recurrent, disabling, and difficultto-treat disease. Several biological events play to develop an autoinflammatory milieu in the skin.

This condition is related with other comorbidities, as insulin resistance and hyperandrogenic states. With the development of new therapies, better knowledge of the pathophysiology, we hope that more physicians will be able to improve the quality of life of patients suffering from HS.

\section{References}

1. Von der Werth JM, Williams HC. The natural history of hidradenitis suppurativa. J Eur Acad Dermatol Venereol. 2000;14(5):389-392.
2. Hunher RE, Laffitte E, Läuchli S, Mainetti C, Mühlstädt M, Schiller P, et al. Swiss Pratice recommendations for the management of hidradenitis suppurative/Acne Inversa. Dermatology. 2017;233(2-3):113-119. doi: 10.1159/000477459

3. Hamzavi IH, Sundaram M, Nicholson C, Zivkovic M, Parks-Miller A, Lee J, et al. Uncovering burden disparity: A comparative analysis of the impact of moderate-to-severe psoriasis and hidradenitis suppurativa. J Am Acad Dermatol. 2017;77(6):1038-1046. doi: 10.1016/j. jaad.2017.07.027

4. Shah A, Alhusayen R, Amini-Nik S. The critical role of macrophages in the pathogenesis of hidradenitis suppurativa. Inflamm Res. 2017;66(11):931-945. doi: 10.1007/s00011-017-1074-y

5. Ralser DJ, Basmanav FB, Tafazzoli A, Wititsuwannakul J, Delker S, Danda $S$, et al. Mutations in $\gamma$-secretase subunit-encoding PSENEN underlie Dowling-Degos disease associated with acne inversa. J Clin Invest. 2017;127(4):1485-1490. doi: 10.1172/JCI90667

6. Zouboulis CC, Del Marmol V, Mrowietz U, Prens EP, Tzellos T, Jemec GB. Hidradenitis Suppurativa/Acne Inversa: Criteria for Diagnosis, Severity Assessment, Classification and Disease Evaluation. Dermatology 2015;231(2):184-190. doi: 10.1159/000431175

7. García-Tutor E, Botellé del Hierro J, San Martín Maya A, Castro García J, España A, Fernández Montero J, et al. Surgical treatment of penile lynphedema associated with hidradenitis suppurativa. Actas Urol Esp. 2005;29(5):519-522.

8. Miller IM, McAndrew RJ, Hamzavi I. Prevalence, Risk Factors, and Comorbidities of Hidradenitis Suppurativa. Dermatol Clin. 2016;34(1):7-16. doi: 10.1016/j.det.2015.08.002

9. Schlapbach C, Hänni T, Yawalkar N, Hunger RE. Expression of the IL23/Th17 pathway in lesions of hidradenitis suppurative. J Am Acad Dermatol. 2011;65(4):790-798. doi: 10.1016/j.jaad.2010.07.010

10. Wolk K, Warszawska K, Hoeflich C, Witte E, Schneider-Burrus S, Witte $\mathrm{K}$, et al. Deficiency of IL-22 contributes to a chronic inflammatory disease: pathogenetic mechanisms in acne inversa. J Immunol. 2011;186(2):1228-1239. doi: 10.4049/jimmunol.0903907

11. Kurokawa I, Nishijima S, Kusumoto K, Senzaki H, Shikata N, Tsubura A. Immunohistochemically study of cytokeratins in hidradenitis suppurativa (acne inversa). J Int Med Res. 2002;30(2):131-136.

12. Ring HC, Thorsen J, Saunte DM, Lilje B, Bay L, Riis PT, et al. The Follicular Skin Microbiome in Patients with Hidradenitis Suppurativa and Healthy Controls. JAMA Dermatol. 2017;153(9):897-905. doi: 10.1001/jamadermatol.2017.0904

13. Ring HC, Bay L, Nilsson M, Kallenbach K, Miller IM, Saunte DM, et al. Bacterial biofilm in chronic lesions of hidradenitis suppurativa. Br J Dermatol. 2017;176(4):993-1000. doi: 10.1111/bjd.15007

14. Hunger RE, Surovy AM, Hassan AS, Braathen LR, Yawalkar N. Toll-like receptor 2 is highly expressed in lesions of acne inversa and colocalizes with C-type lectin receptor. Br J Dermatol. 2008;158(4):691-697. doi: 10.1111/j.1365-2133.2007.08425.x

15. Emelianov VU, Bechara FG, Gläser R, Langan EA, Taungjaruwinai WM, Schröder JM, et al. Immunohistological pointers to a possible role for excessive cathelicidin (LL-37) expression by apocrine sweat glands in the pathogenesis of hidradenitis suppurativa/acne inversa. $\mathrm{Br} J$ Dermatol. 2012;166(5):1023-1034. doi: 10.1111/j.13652133.2011.10765.x

16. Napolitano M, Megna M, Timoshchuk EA, Patruno C, Balato N, Fabbrocini G, et al. Hidradenitis suppurativa: from pathogenesis to diagnosis and treatment. Clin Cosmet Investig Dermatol. 2017;10:105115. doi: 10.2147/CCID.S111019 
17. Jang Y, Lee AY, Jeong SH, Park KH, Paik MK, Cho NJ, et al. Chlorpyrifos induces NLRP3 inflammasome and pyroptosis/apoptosis via mitochondrial oxidative stress in human keratinocyte HaCaT cells Toxicology. 2015;338:37-46. doi: 10.1016/j.tox.2015.09.006

18. Di Paolo NC, Shayakhmetov DM. Interleukin $1 \alpha$ and the inflammatory process. Nature immunology. 2016;17(8):906-913. doi: 10.1038/ ni.3503

19.Jorch SK, Kubes P. An emerging role for neutrophil extracellular traps in noninfectious disease. Nat Med. 2017;23(3):279-287. doi: 10.1038/ nm. 4294

20. Matusiak Ł, Szczęch J, Bieniek A, Nowicka-Suszko D, Szepietowski JC. Increased interleukin (IL)-17 serum levels in patients with hidradenitis suppurativa: Implications for treatment with anti-IL-17 agents. J Am Acad Dermatol. 2017;76(4):670-675. doi: 10.1016/j. jaad.2016.10.042

21. Winer S, Paltser G, Chan Y, Tsui H, Engleman E, Winer D, Dosch HM Obesity predisposes to Th17 bias. Eur J Immunol. 2009;39(9):26292635. doi: $10.1002 /$ eji.200838893

22. Montalbano AM, Riccobono L, Siena L, Chiappara G, Di Sano C, Anzalone G, et al. Profita Cigarette smoke affects IL-17A, IL-17F and IL17 receptor expression in the lung tissue: Ex vivo and in vitro studies. Cytokine. 2015;76(2):391-402. doi: 10.1016/j.cyto.2015.07.013

23.Sumarac-Dumanovic M, Stevanovic D, Ljubic A, Jorga J, Simic M Stamenkovic-Pejkovic D, et al. Increased activity of interleukin-23/ interleukin-17 proinflammatory axis in obese women. Int J Obes (Lond). 2009;33(1):151-156. doi: 10.1038/ijo.2008.216

24. Karakose M, Demircan K, Tutal E, Demirci T, Arslan MS, Sahin M, et al. Clinical significance of ADAMTS1, ADAMTS5, ADAMTS9 aggrecanases and IL-17A, IL-23, IL-33 cytokines in polycystic ovary syndrome. J Endocrinol Invest. 2016;39(11):1269-1275.

25. Randhawa HK, Hamilton J, Pope E. Finasteride for the Treatment of Hidradenitis Suppurativa in Children, and Adolescents. JAMA Dermatol. 2013;149(6):732-735. doi: 10.1001/jamadermatol.2013.2874

26.Zouboulis CC, Desai N, Emtestam L, Hunger RE, Ioannides D, Juhász I, et al. European S1 guideline for the treatment of hidradenitis suppurativa/acne inversa. J Eur Acad Dermatol Venereol. 2015;29(4):619-644. doi: 10.1111/jdv.12966

27. Hessam S, Sand M, Gambichler T, Skrygan M, Rüddel I, Bechara FG. IL-36 in hidradenitis suppurativa: Evidence for a distinctive pro-inflammatory role and a key factor in the development of an inflammatory loop. Br J Dermatol. 2017.

28. Boutet M-A, Bart G, Penhoat M, Amiaud J, Brulin B, Charrier C, et al. Distinct expression of interleukin (IL)-36 $\alpha, \beta$ and $\gamma$, their antagonist IL-36Ra and IL-38 in psoriasis, rheumatoid arthritis, and Crohn's disease. Clin Exp Immunol. 2016;184(2):159-173. doi: 10.1111/ cei.12761

29. Nishida A, Hidaka K, Kanda T, Imaeda H, Shioya M, Inatomi O, et al. Increased Expression of Interleukin-36, a Member of the Interleukin-1 Cytokine Family, in Inflammatory Bowel Disease. Inflamm Bowel Dis 2016;22(2):303-314. doi: 10.1097/MIB.0000000000000654

30. Johnston A, Xing X, Guzman AM, Riblett M, Loyd CM, Ward NL, et al. IL-1F5, -F6, -F8, and -F9: a novel IL-1 family signaling system that is active in psoriasis and promotes keratinocyte antimicrobial peptide expression. J Immunol. 2011;186(4):2613-2622.

31. Di Caprio R, Balato A, Caiazzo G, Lembo S, Raimondo A, Fabbrocini G, et al. IL-36 cytokines are increased in acne and hidradenitis suppurativa. Arch Dermatol Res. 2017;309(8):673-678. doi: 10.1007/s00403-017$1769-5$
32. Kanni T, Zenker O, Habel M, Riedemann N, Giamarellos-Bourboulis EJ. Complement activation in hidradenitis suppurativa: a new pathway of pathogenesis? Br J Dermatol. 2018. doi: 10.1111/bjd.16428

33. Burney RE. 35-Year Experience with Surgical Treatment of Hidradenitis Suppurativa. World J Surg. 2017;41(11):2723-2730. doi: 10.1007/ s00268-017-4091-7

34. Kim ES, Garnock-Jones KP, Keam SJ. Adalimumab: A Review in Hidradenitis Suppurativa. Am J Clin Dermatol. 2016;17(5):545-552.

35. Martorell A, Caballero A, González Lama Y, Jiménez-Gallo D, Lázaro Serrano M, et al. Management of patients with hidradenitis suppurativa. Actas Dermosifiliogr. 2016;107 Suppl 2:32-42. doi: 10.1016/S0001-7310(17)30007-8

36. Grant A, Gonzalez T, Montgomery MO, Cardenas V, Kerdel FA. Infliximab therapy for patients with moderate to severe hidradenitis suppurativa: a randomized, double-blind, placebo-controlled crossover trial. J Am Acad Dermatol. 2010;62(2):205-217. doi: 10.1016/j.jaad.2009.06.050

37. Lee RA, Dommasch E, Treat J, Sciacca-Kirby J, Chachkin S, Williams J, et al. A prospective clinical trial of open-label etanercept for the treatment of hidradenitis suppurativa. J Am Acad Dermatol. 2009;60(4):565573. doi: 10.1016/j.jaad.2008.11.898

38. Maarouf M, Clark AK, Lee DE, Shi VY. Targeted treatments for hidradenitis suppurativa: a review of the current literature and ongoing clinical trials. J Dermatolog Treat. 2017:1-9. doi: $10.1080 / 09546634.2017$

39. Blok JL, Li K, Brodmerkel C, Horvatovich P, Jonkman MF, Horvath B. Ustekinumab in hidradenitis suppurativa: clinical results and a search for potential biomarkers in serum. Br J Dermatol. 2016;174(4):839846. doi: $10.1111 /$ bjd.14338

40. Tonini A, Gualtieri B, Panduri S, Romanelli M, Chiricozzi A. A new class of therapeutic biologic agents for combatting psoriasis: anti-IL-23 agents. Expert Opin Biol Ther. 2017:1-14.

41. Tzanetakou V, Kanni T, Giatrakou S, Katoulis A, Papadavid E, Netea MG, et al. Safety and Efficacy of Anakinra in Severe Hidradenitis Suppurativa: A Randomized Clinical Trial. JAMA Dermatol. 2016;152(1):52-59. doi: 10.1001/jamadermatol.2015.3903

42. Russo V, Alikhan A. Failure of Anakinra in a Case of Severe Hidradenitis Suppurativa. J Drugs Dermatol. 2016;15(6):772-774.

43. Houriet C, Seyed Jafari SM, Thomi R, Schlapbach C, Borradori L, Yawalkar N, et al. Canakinumab for Severe Hidradenitis Suppurativa: Preliminary Experience in 2 Cases. JAMA Dermatol. 2017;153(11):1195-1197. doi: 10.1001/jamadermatol.2017.2392

44. Kanni T, Argyropoulou M, Spyridopoulos T, Pistiki A, Stecher M, Dinarello CA, et al. MABp1 Targeting Interleukin-1Alpha for Moderate to Severe Hidradenitis Suppurativa not Eligible for Adalimumab: A Randomized Study. J Invest Dermatol. 2018;138(4):795-801. doi: 10.1016/j.jid.2017.10.030

45. Thorlacius L, Theut Riis P, Jemec GBE. Severe hidradenitis suppurativa responding to treatment with secukinumab: a case report. Br J Dermatol. 2017. doi: 10.1111/bjd.15769.

46. Schuch A, Fischer T, Boehner A, Biedermann T, Volz T. Successful Treatment of Severe Recalcitrant Hidradenitis Suppurativa with the Interleukin-17A Antibody Secukinumab. Acta Derm Venereol. 2018;98(1):151-152. doi: 10.2340/00015555-2794.

47. ClinicalTrials.gov. Bethesda (MD): National Library of Medicine (US). Identifier NCT03099980. Exploratory Trial Evaluating Cosentyx (Secukinumab) for Patients with Moderate-to-Severe Hidradenitis 
Suppurativa. Available from: https://clinicaltrials.gov/ct2/show/ NCT03099980

48. ClinicalTrials.gov. Bethesda (MD): National Library of Medicine (US). Identifier NCT03248531. A Study to Test the Efficacy, Safety and Pharmacokinetics of Bimekizumab in Subjects with Moderate to Severe Hidradenitis suppurativa. Available from: https://clinicaltrials. gov/ct2/show/NCT03248531

49. ClinicalTrials.gov. Bethesda (MD): National Library of Medicine (US). Identifier NCT02421172.Efficacy, Safety, and Pharmacokinetics Study of CJM112 in Hidradenitis Suppurativa Patients. Available from: https://clinicaltrials.gov/ct2/show/NCT02421172
50. Jennings L, Nestor L, Molloy O, Hughes R, Moriarty B, Kirby B. The treatment of hidradenitis suppurativa with the glucagon-like peptide-1 agonist liraglutide. Br J Dermatol. 2017;177(3):858-859. doi: 10.1111/bjd.15233

51. Mahil SK, Catapano M, Di Meglio P, Dand N, Ahlfors H, Carr IM, et al. An analysis of IL-36 signature genes and individuals with IL1RL2 knockout mutations validates IL-36 as a psoriasis therapeutic target. Sci Transl Med. 2017;9(411). pii: eaan2514. doi: 10.1126/ scitranslmed.aan2514

52. IFX-1 blocking the anaphylatoxin 1 blocking the anaphylatoxin 1 blocking the anaphylatoxin $\mathrm{C} 5 \mathrm{a}-$ an anti an anti-inflammatory effect in inflammatory effect in patients with hidradenitis suppurativa. Available from: www.inflarx.com/dam/jcr:ec982d1e.../Guo_2017_ poster.pdf 